\title{
Antijamming Performance of Adaptive Chaos Based CDMA System with MRC in Imperfect Channel Estimation Environment
}

\author{
Meher Krishna Patel, Stevan M. Berber, and Kevin W. Sowerby \\ The University of Auckland, Auckland, New Zealand \\ Correspondence should be addressed to Meher Krishna Patel; mpat260@aucklanduni.ac.nz
}

Received 5 July 2016; Accepted 14 September 2016; Published 10 January 2017

Academic Editor: Enrico M. Vitucci

Copyright (C) 2017 Meher Krishna Patel et al. This is an open access article distributed under the Creative Commons Attribution License, which permits unrestricted use, distribution, and reproduction in any medium, provided the original work is properly cited.

This paper presents the antijamming performance of adaptive multiuser DS-CDMA system using maximal ratio combining technique in slow switching pulse noise jammer. Complex flat fading channel coefficients are assumed to be unknown at the receiver. Pilot symbols are used to estimate the fading coefficients using least mean square (LMS) algorithm, and effect of imperfect channel estimation on bit error rate (BER) is studied. Under the perfect synchronization conditions, probability of error in closed form is derived.

\section{Introduction}

Fading is the phenomena which makes the wireless communication difficult as compared to other systems, for example, optical communication and coaxial cable communication. Diversity, channel estimation algorithms, and spread spectrum techniques can be used to mitigate its effect without increasing the transmission power [1].

Increasing number of users is a challenge in communication systems. Code division multiple access (CDMA) is the spread spectrum technique which allows multiple users to share the complete bandwidth. In CDMA system, each user is distinguished by their spread sequence. There are various types of spreading sequence that are available, for example, pseudorandom noise (PN) sequences and chaotic sequences. Comparison between chaos based and $\mathrm{PN}$ sequence based CDMA system is shown in $[2,3]$. Due to noise-like feature of chaotic sequences, chaos based CDMA systems gain interest among researchers [4-9].

Jammer is the device which can block the desired transmitted signal of any frequency range [10]. Among the various security attacks, jammer is the simplest; therefore, antijamming performances are widely studied in the literature. Antijamming performances of chaos based CDMA system are shown in the presence of Gaussian noise in [11, 12]. Further, it is studied for flat fading channels with real coefficients in [13]. Performance of such systems will severely degrade in the presence complex fading coefficients.

In diversity technique copies of a signal are sent multiple times. Among the various forms of diversities antenna diversity is the widely used technique. Choosing the best copy among the received signals is not an optimal solution; therefore, all the copies of received signals are combined in maximal ratio combining (MRC) technique. Since all the received signals should be in same phase for MRC; therefore, channel estimators play important role in MRC technique. Performance of antenna diversity techniques for chaos based CDMA system in various environments is studied in [14]. The role of channel estimator in MRC technique is shown in [1]. Further, diversity techniques in jamming environment for chaos based CDMA system with adaptive estimator are never studied.

The objective of this paper is to investigate the antijamming performance of chaos based CDMA system with imperfect channel estimation in complex flat fading channel for downlink systems. MRC technique is used to improve the performance of the system and BER expression in closed form is derived for BPSK system for slowly time varying 
channel. Moreover, it is shown analytically that, in complex fading environment, only real part of the fading coefficient affects the BER performance of the system. Also, it is proved mathematically that the estimated amplitude coefficient has no effect on the BER performance for single antenna system. Further, the work presented in [12] is shown as the special cases of this work.

This paper is organized as follows. Chaos based adaptive CDMA system with MRC technique in jamming environment is shown in Section 2. Section 3 presents the analytical performance of the system. Simulation results are shown in Section 4. Concluding remarks are given in Section 5.

\section{System Model}

Baseband representation of the chaos based CDMA system is shown in Figure 1 . The baseband signal $s_{k}^{n}$ for $n$th user at $k$ th chip instant is given by

$$
s_{k}^{n}=\gamma_{i}^{n} x_{k}^{n}
$$

where $\gamma_{i}^{n}$ is $n$th user symbol at $i$ th time instant, $x_{k}^{n}$ is $k$ th chip of $n$th user chaotic spreading sequence or chip within an information bit (i.e., $k=1, \ldots, 2 \beta$ ), and $2 \beta$ is the spreading factor. Chip energy and bit energy are denoted by $P_{c}$ and $E_{b}$, respectively. For simplicity of expressions, $n=0,1$ are used to denote the desired user symbols and pilot symbols, receptively.

In jamming environment, $k$ th received chip $r_{k}^{0}$ of $i$ th symbols for desired user is given by

$$
r_{k, m}^{0}=\sum_{n=0}^{N} s_{k}^{n} h_{i, m}+\xi_{k, m}+\zeta_{k, m}
$$

where subscript $m$ denotes the signals corresponding to path $m=1,2, \ldots, M$ and $\xi_{k}$ is $k$ th chip of complex additive white Gaussian noise with power spectral density equal to $N_{0}$. Pulsed noise jamming signal is denoted by $\zeta_{k}$ and modeled using zero mean Gaussian noise with variance equal to $P_{j}$ and duty cycle $\rho$ [12]. The complex channel coefficient at $i$ th time instant is denoted by $h_{i}=a_{i} e^{j \phi_{i}}$, where $a_{i}$ and $\phi_{i}$ amplitude distortion and phase distortion are caused by the channel, respectively.

\section{Receiver Performance}

Receiver structure and LMS estimation algorithm are exactly the same as shown in [1] with one multipath that is $L=$ 1 in each channel. Putting $L=1$ and including the jamming signal there, the decision variable can be represented as

$$
Z_{i}=A+B+C+D
$$

where

$$
\begin{aligned}
& \underbrace{A=\sum_{m=1}^{M} a_{i, m} \widehat{a}_{i, m} \cos \left(\phi_{i, m}-\widehat{\phi}_{i, m}\right) \gamma_{i}^{0} \sum_{k=1}^{2 \beta}\left(x_{k}^{0}\right)^{2}}_{\text {Desired Signal }} \\
& \underbrace{B=\sum_{m=1}^{M} a_{i, m} \widehat{a}_{i, m} \cos \left(\phi_{i, m}-\widehat{\phi}_{i, m}\right) \sum_{n=1}^{N} \sum_{k=1}^{2 \beta} \gamma_{i}^{n} x_{k}^{n} x_{k}^{0}}_{\text {Interuser Interference }} \\
& \underbrace{C=\sum_{m=1}^{M} \widehat{a}_{i, m} \cos \left(\hat{\phi}_{i, m}\right) \sum_{k=1}^{2 \beta} \operatorname{Re}\left\{\xi_{k, m}\right\} x_{k}^{0}+\sum_{m=1}^{M} \widehat{a}_{i, m} \sin \left(\hat{\phi}_{i, m}\right) \sum_{k=1}^{2 \beta} \operatorname{Im}\left\{\xi_{k, m}\right\} x_{k}^{0}}_{\text {Noise }} \\
& \underbrace{D=\sum_{m=1}^{M} \widehat{a}_{i, m} \cos \left(\widehat{\phi}_{i, m}\right) \sum_{k=1}^{2 \beta} \operatorname{Re}\left\{\zeta_{k, m}\right\} x_{k}^{0}+\sum_{m=1}^{M} \widehat{a}_{i, m} \sin \left(\widehat{\phi}_{i, m}\right) \sum_{k=1}^{2 \beta} \operatorname{Im}\left\{\zeta_{k, m}\right\} x_{k}^{0},}_{\text {Jamming Signal }}
\end{aligned}
$$

where $\operatorname{Re}(\cdot)$ and $\operatorname{Im}(\cdot)$ are the real and imaginary parts of signals. Sign of this decision variable is the estimated symbol; that is,

$$
\widehat{\gamma}_{i}^{0}=\operatorname{sign}\left(Z_{i}\right) .
$$

The probability of error $P\left(a_{i}\right)$ for $i$ th bit of desired user is given by [12]

$$
P\left(a_{i}\right)=P_{\text {Jammer On }}\left(a_{i}\right)+P_{\text {Jammer Off }}\left(a_{i}\right) .
$$

$P_{\text {Jammer On }}\left(a_{i}\right)$ and $P_{\text {Jammer Off }}\left(a_{i}\right)$ are the probability of error when jammer is on and off, respectively, and given by

$$
\begin{aligned}
& P_{\text {Jammer On }}\left(a_{i}\right)=\frac{\rho}{2} \\
& \quad \operatorname{erfc}\left(\frac{E\left[Z_{i}^{g} \mid \gamma_{i}^{g}=1\right]}{\sqrt{2 \operatorname{var}(A)+2 \operatorname{var}(B)+2 \operatorname{var}(C)+2 \operatorname{var}(D)}}\right)
\end{aligned}
$$




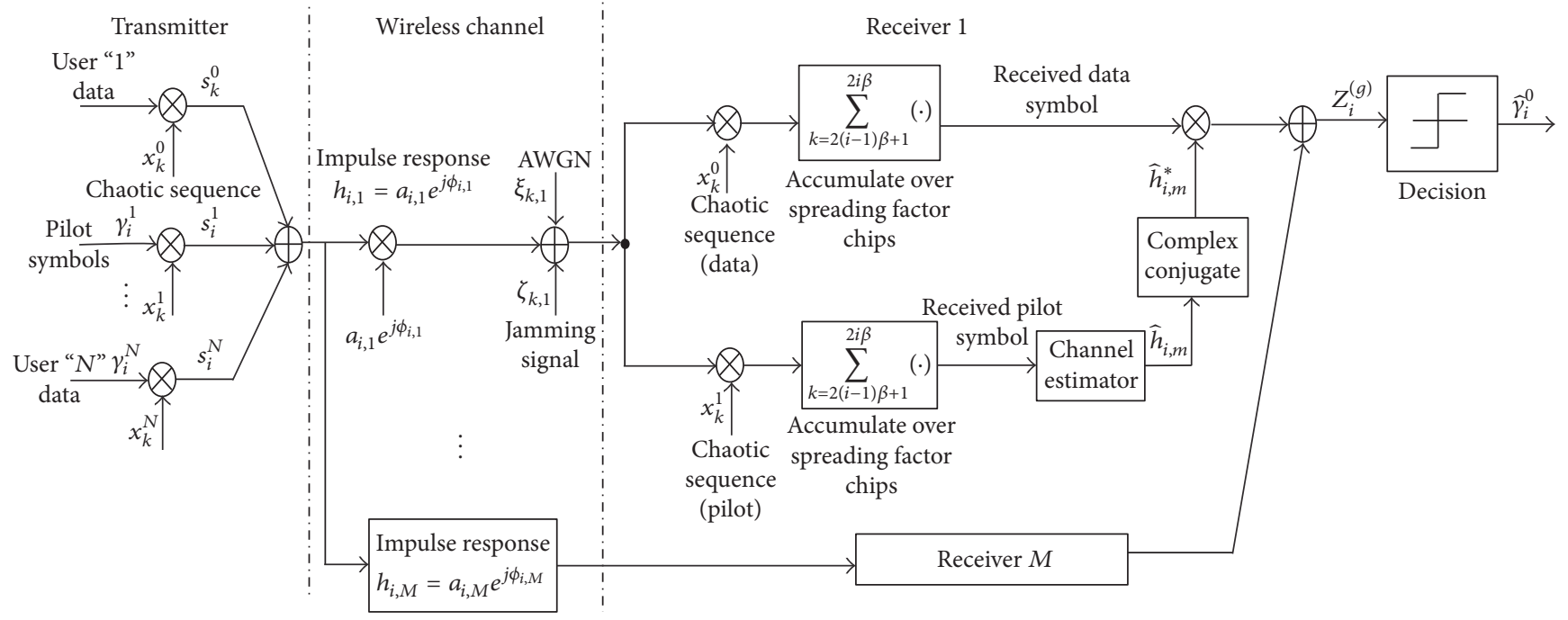

FIGURE 1: Block diagram of proposed system.

$$
\begin{aligned}
& P_{\text {Jammer Off }}\left(a_{i}\right)=\frac{1-\rho}{2} \\
& \quad \operatorname{erfc}\left(\frac{E\left[Z_{i}^{g} \mid \gamma_{i}^{g}=1\right]}{\sqrt{2 \operatorname{var}(A)+2 \operatorname{var}(B)+2 \operatorname{var}(C)}}\right) .
\end{aligned}
$$

Variances of (6) and (7) are same except variance of AWGN; that is, $N_{0}$ is replaced by $\rho P_{j}$ in jamming signal case. Putting $L=1$ and neglecting intersymbol interference term in appendix $\mathrm{B}$ of [1], we have the following probability of equation:

$$
\begin{aligned}
P\left(a_{i}\right)= & \frac{\rho}{2} \operatorname{erfc}(W+X+Y+Z)^{-1 / 2} \\
& +\frac{1-\rho}{2} \operatorname{erfc}(W+X+Y)^{-1 / 2},
\end{aligned}
$$

where

$$
\begin{aligned}
& W=\frac{\operatorname{var}\left\{\left(x_{k}^{0}\right)^{2}\right\}}{\beta P_{c}^{2}} \\
& X=\frac{N}{\beta} \\
& Y=\left[\frac{\left\{\sum_{m=1}^{M} a_{i, m} \widehat{a}_{i, m} \cos \left(\phi_{i, m}-\widehat{\phi}_{i, m}\right)\right\}^{2}}{\sum_{m=1}^{M} \widehat{a}_{i, m}^{2}} \frac{E_{b}}{N_{0}}\right]^{-1} \\
& Z=\left[\frac{\left\{\sum_{m=1}^{M} a_{i, m} \widehat{a}_{i, m} \cos \left(\phi_{i, m}-\widehat{\phi}_{i, m}\right)\right\}^{2}}{\rho \sum_{m=1}^{M} \hat{a}_{i, m}^{2}} \frac{E_{b}}{P_{j}}\right]^{-1} .
\end{aligned}
$$

Hence, the probability of error depends on the number of users $(N)$, spreading factor $(2 \beta)$, estimated channel coefficients $\left(\widehat{a}_{m}, \widehat{\phi}_{m}\right)$, the signal to noise ratio $\left(E_{b} / N_{0}\right)$, duty cycle of pulse noise jammer $(\rho)$, signal to jamming power ratio $\left(E_{b} / P_{j}\right)$, and number of receiving antennas $(M)$. Clearly probability of error increases as the number of users and duty cycle of jamming power increase in the system. Further, probability of error decreases as the spreading factor, signal to noise ratio, signal to jamming power ratio, and/or number of receiving antenna increase.

Also, the error probability depends on the difference between estimated phase and actual phase of the complex channel coefficients. As the estimated phase error $\left(\phi_{i}-\right.$ $\widehat{\phi}_{i}$ ) decreases, value of cosine function increases. Therefore, overall value of SNR $\left(E_{b} / N_{0}\right)$ and signal to jamming power ratio $\left(E_{b} / P_{j}\right)$ increase. Hence, the performance of the system is greatly dependent on the performance of the channel estimation algorithms. Further, it can be seen that only real parts of the complex fading coefficients appear in the BER equation, that is, $a_{i} \cos \left(\phi_{i}\right)$; that is, imaginary parts of the fading coefficients $a_{i} \sin \left(\phi_{i}\right)$ do not affect the system. Also, for one antenna system, that is, $m=1$, the estimated amplitude value will disappear from the equation which shows that the estimated amplitude coefficient has no effect on the BER performance for single antenna system.

For perfect channel estimator, that is, $\widehat{a}_{i, m} \cos \left(\widehat{\phi}_{i, m}\right)=$ $a_{i, m} \cos \left(\phi_{i, m}\right),(12)$ reduces to

$$
\begin{gathered}
P_{\text {perfect }}\left(a_{i}\right)=\frac{\rho}{2} \operatorname{erfc}\left(\frac{\operatorname{var}\left\{\left(x_{k}^{0}\right)^{2}\right\}}{\beta P_{c}^{2}}+\frac{N}{\beta}\right. \\
\left.+\left[\sum_{m=1}^{M} a_{i, m}^{2} \frac{E_{b}}{N_{0}}\right]^{-1}+\left[\sum_{m=1}^{M} a_{i, m}^{2} \frac{E_{b}}{\rho P_{j}}\right]^{-1}\right)^{-1 / 2} \\
+\frac{1-\rho}{2} \operatorname{erfc}\left(\frac{\operatorname{var}\left\{\left(x_{k}^{0}\right)^{2}\right\}}{\beta P_{c}^{2}}+\frac{N}{\beta}\right. \\
\left.+\left[\sum_{m=1}^{M} a_{i, m}^{2} \frac{E_{b}}{N_{0}}\right]^{-1}\right)^{-1 / 2} .
\end{gathered}
$$




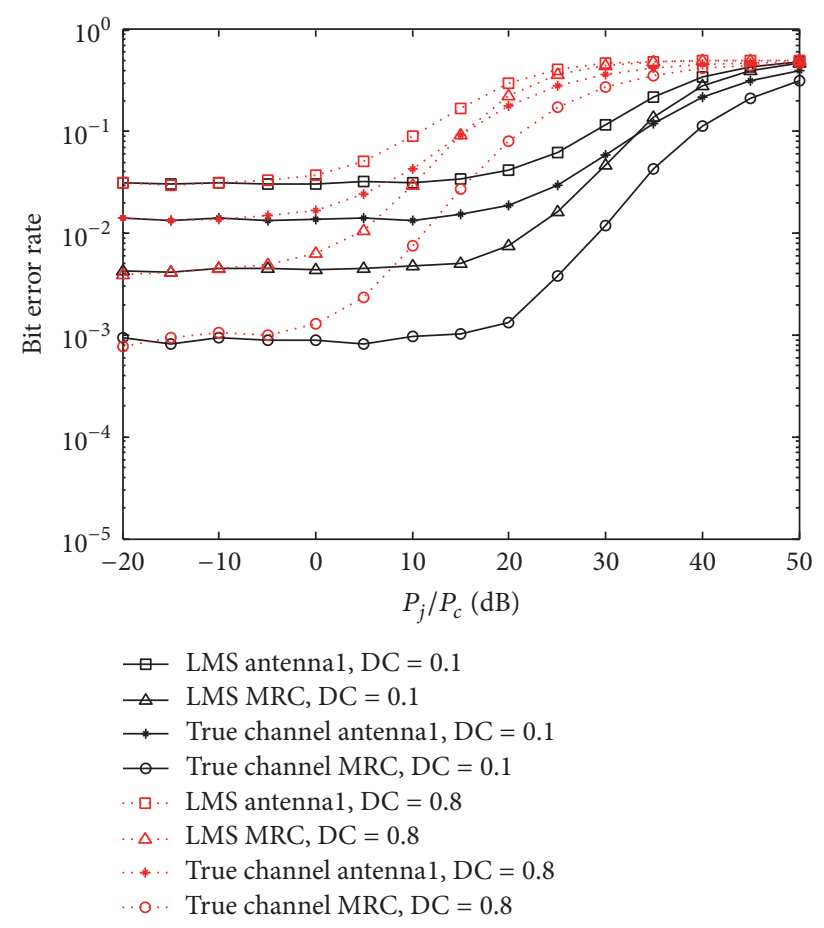

FIGURE 2: $P_{j} / P_{c}$ versus BER: performance degradation with increase in duty cycle (DC) of jamming signal, $\mathrm{SF}=50, \mathrm{SNR}=20 \mathrm{~dB}$, and User $=2$.

Further, if we assume single user system with no pilot symbols and $h=1 e^{0}$, that is, no fading environment, then we can remove the estimator part from Figure 1 ; that is, $\widehat{h}=1 e^{0}$. In this case, the above equation reduces to expression derived in [12].

In general, the BER of system $P_{e}$ can be expressed as

$$
P_{e}=\int_{0}^{\infty} P\left(a_{i}\right) p(a) d a,
$$

where the subscript $p(a)$ is the joint probability density function of true and estimated channel coefficients. Since estimated channel coefficients do not have any well-defined probability density function, therefore, it is difficult to integrate the above equation $[2,15]$.

\section{Simulation Results}

Various simulation results are shown to demonstrate the performance of the proposed system. In simulation results, "LMS" and "True" represent the bit error rate performances of the system using LMS estimator and perfect channel estimation case. "Antennal" and "MRC" represent the performance of single antenna and MRC technique, respectively. Similarly, "DC" and "SF" represent duty cycle of jamming signal and spreading factor, receptively.

$P_{j} / P_{c}$ versus BER performances of the system are shown in Figures 2 and 3. Firstly, Figure 2 shows the significant performance improvement in the system using MRC techniques. Secondly, it shows the performance degradation in the system with increase in the duty cycle of the jamming signal. In

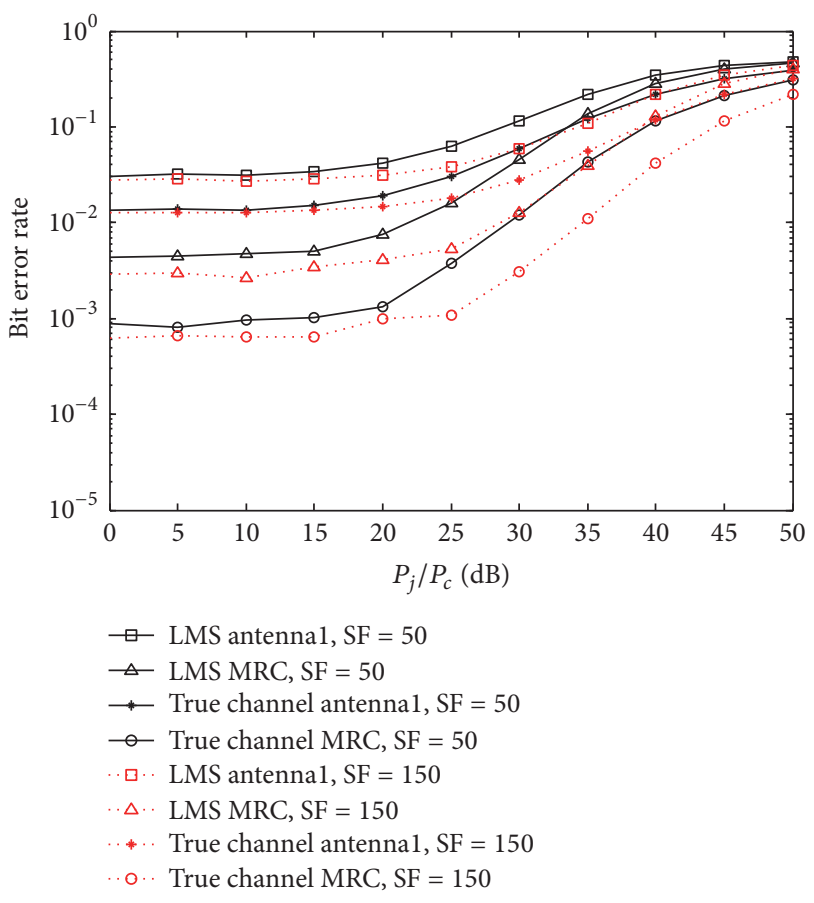

FIGURE 3: $P_{j} / P_{c}$ versus BER: performance improvement with increase in spreading factor, $\mathrm{DC}=0.1, \mathrm{SNR}=20 \mathrm{~dB}$, and User $=2$.

the other words, if duty cycle of jammer is high, then it will affect larger number of chips in the signal. Since, performance of decorrelator in CDMA system depends on the spreading sequence, therefore disruption in the chip sequences severely degrades the BER performance of the system as shown in the figure. Further effect of duty cycle is negligible for low $P_{j} / P_{c}$ values, that is, from $0 \mathrm{~dB}$ to $-20 \mathrm{~dB}$ SNR; that is, jammer power is not sufficient to disrupt the signal.

Equation (12) shows that the spreading factor $\beta$ reduces the terms $W$ and $X$, which are generated by other users' signals. It is verified by the results in Figure 3, which shows the performance improvement in the system with increase in spreading factor. Also, the performance degradation due to duty cycle of jamming signal can be compensated by increasing the spreading factor.

$E_{b} / N_{0}$ versus BER performances of the system are shown in Figures 4 and 5. From these two figures it is clear that BER performance decreased with increase in number of users and duty cycle of the jamming signals. With increase in number of users in the system, the interuser interference increases which results in degradation in the BER as shown in Figure 4. Further, Figure 5 is another way for representing the effect of duty cycle in the proposed system, and explanation is the same as that given for Figure 2. In all the simulations results, the performance differences can be between LMS estimator and true estimator can be minimized using good estimation algorithms.

\section{Conclusion}

In this paper the antijamming performances of chaos based CDMA system with MRC technique are evaluated in flat 


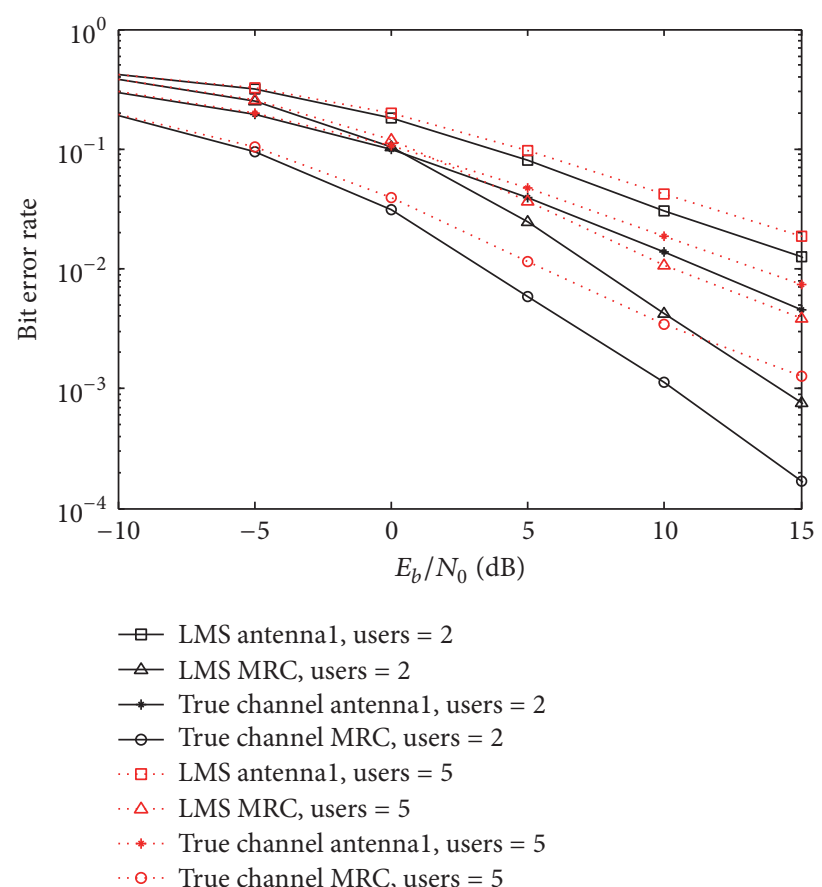

FIGURE 4: $E_{b} / N_{0}$ versus BER: performance degradation with increase in number of users in the system, $\mathrm{DC}=0.1, \mathrm{SF}=50$, and $P_{j} / P_{c}=10 \mathrm{~dB}$.

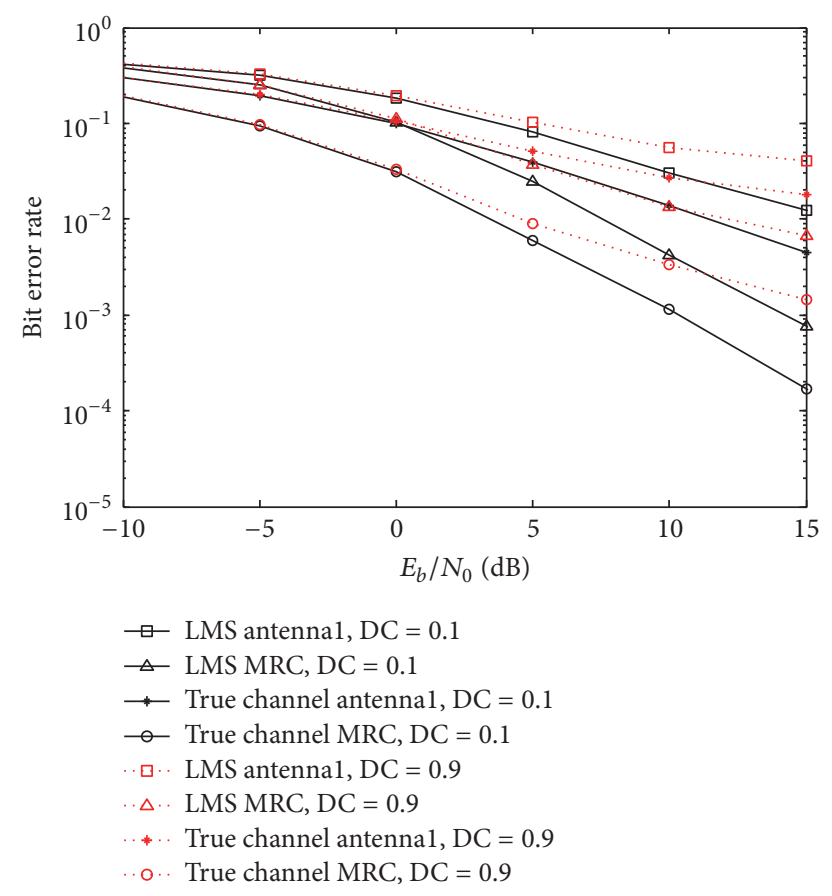

FIGURE 5: $E_{b} / N_{0}$ versus BER: performance degradation with increase in duty cycle of jamming signal, $\mathrm{SF}=50, P_{j} / P_{c}=20 \mathrm{~dB}$, and User $=1$.

fading channel. Performance improvement in the system using MRC technique is shown analytically. Effects of jamming powers, spreading factors, and number of users are shown. Further, performance of LMS channel estimator is compared with perfect channel estimators in the simulation results.

\section{Competing Interests}

The authors declare that they have no competing interests.

\section{References}

[1] M. K. Patel, S. M. Berber, and K. W. Sowerby, "Performance analysis of adaptive chaos based CDMA system with antenna diversity in frequency selective channel," Wireless Personal Communications, vol. 84, no. 2, pp. 1439-1448, 2015.

[2] M. K. Patel, S. M. Berber, and K. W. Sowerby, "Adaptive RAKE receiver in chaos based pilot-added DS-CDMA system," Physical Communication, vol. 16, pp. 37-42, 2015.

[3] G. Heidari-Bateni and C. McGillem, "Chaotic sequences for spread spectrum: an alternative to pn-sequences," in Proceedings of the IEEE International Conference on Selected Topics in Wireless Communications, pp. 437-440, IEEE, 1992.

[4] X. Shu, H. Wang, and J. Wang, "Underwater chaos-based DSCDMA system," in Proceedings of the IEEE International Conference on Signal Processing, Communications and Computing (ICSPCC '11), pp. 1-6, IEEE, Xi'an, China, September 2011.

[5] B. Jovic, "Chaos-based BPSK communication system," Electronics Letters, vol. 51, no. 8, pp. 630-632, 2015.

[6] G. Kaddoum and F. Shokraneh, "Analog network coding for multi-user multi-carrier differential chaos shift keying communication system," IEEE Transactions on Wireless Communications, vol. 14, no. 3, pp. 1492-1505, 2015.

[7] S. M. Berber, "Probability of error derivatives for binary and chaos-based CDMA systems in wide-band channels," IEEE Transactions on Wireless Communications, vol. 13, no. 10, pp. 5596-5606, 2014.

[8] G. Kaddoum, "Wireless chaos-based communication systems: a comprehensive survey," IEEE Access, vol. 4, pp. 2621-2648, 2016.

[9] A. Tayebi, S. Berber, and A. Swain, "Performance analysis of chaotic DSSS-CDMA synchronization under jamming attack," Circuits, Systems, and Signal Processing, vol. 35, no. 12, pp. 43504371, 2016.

[10] Y.-S. Shiu, S. Y. Chang, H.-C. Wu, S. C.-H. Huang, and H.-H. Chen, "Physical layer security in wireless networks: a tutorial," IEEE Wireless Communications, vol. 18, no. 2, pp. 66-74, 2011.

[11] F. C. M. Lau, M. Ye, C. K. Tse, and S. F. Hau, "Anti-jamming performance of chaotic digital communication systems," IEEE Transactions on Circuits and Systems I: Fundamental Theory and Applications, vol. 49, no. 10, pp. 1486-1494, 2002.

[12] F. C. M. Lau and C. K. Tse, "Performance of chaos-based digital communication systems in the presence of a pulsednoise jammer," Circuits, Systems, and Signal Processing, vol. 23, no. 3, pp. 169-194, 2004.

[13] A. Tayebi, S. Berber, and A. Swain, "Performance analysis of synchronization in chaotic DSSS-CDMA system under jamming attack," in Proceedings of the International Symposium on Information Theory and Its Applications (ISITA '14), Melbourne, Australia, October 2014.

[14] L. F. Abdulameer, J. D. Jignesh, U. Sripati, and M. Kulkarni, "BER performance enhancement for secure wireless optical communication systems based on chaotic MIMO techniques," Nonlinear Dynamics, vol. 75, no. 1-2, pp. 7-16, 2014.

[15] G. Kaddoum, M. Coulon, D. Roviras, and P. Chargé, "Theoretical performance for asynchronous multi-user chaos-based communication systems on fading channels," Signal Processing, vol. 90, no. 11, pp. 2923-2933, 2010. 


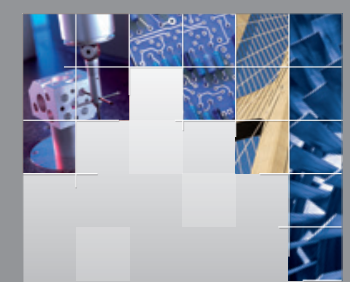

\section{Enfincering}
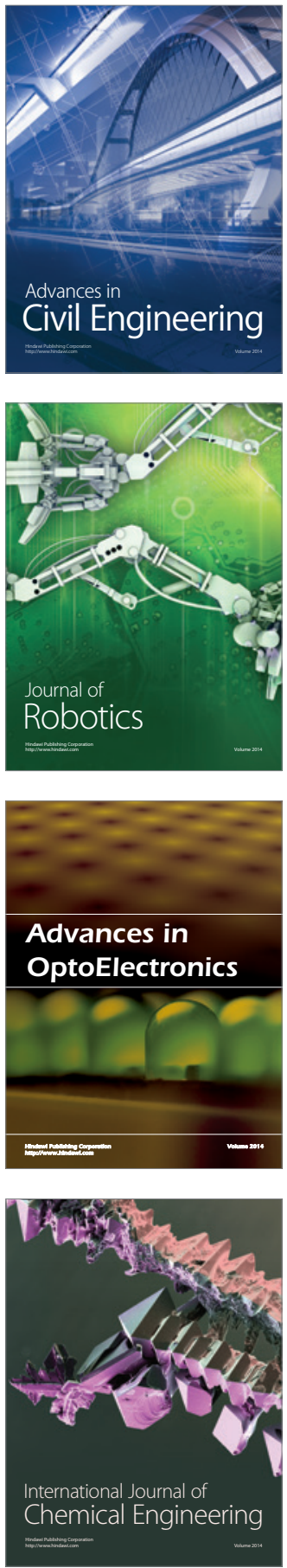

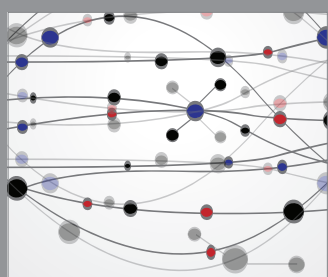

The Scientific World Journal

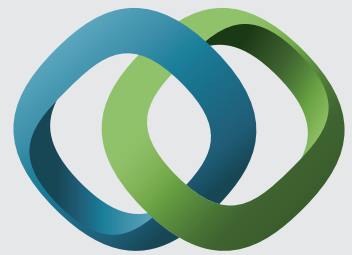

\section{Hindawi}

Submit your manuscripts at

https://www.hindawi.com
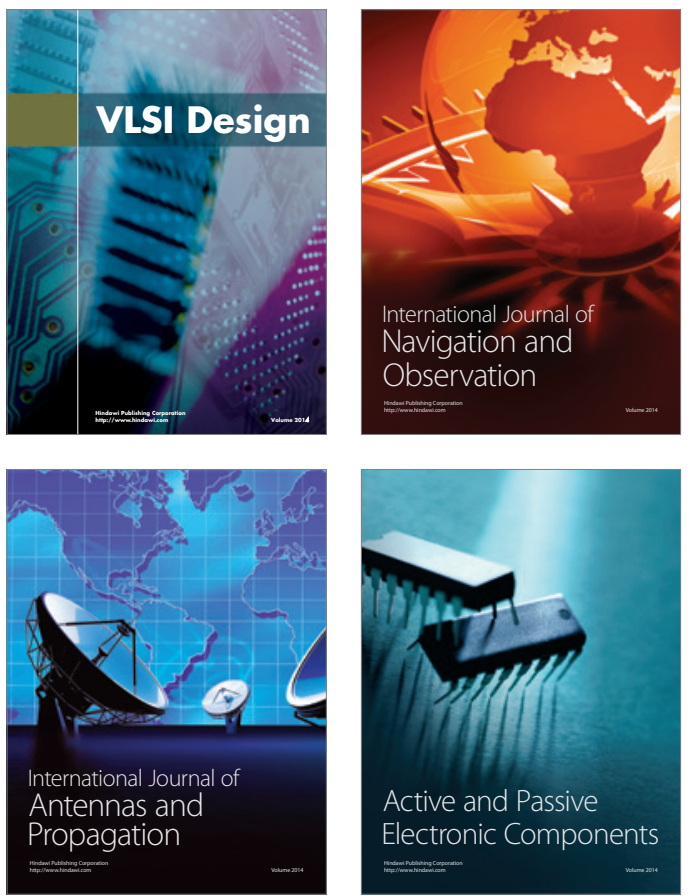
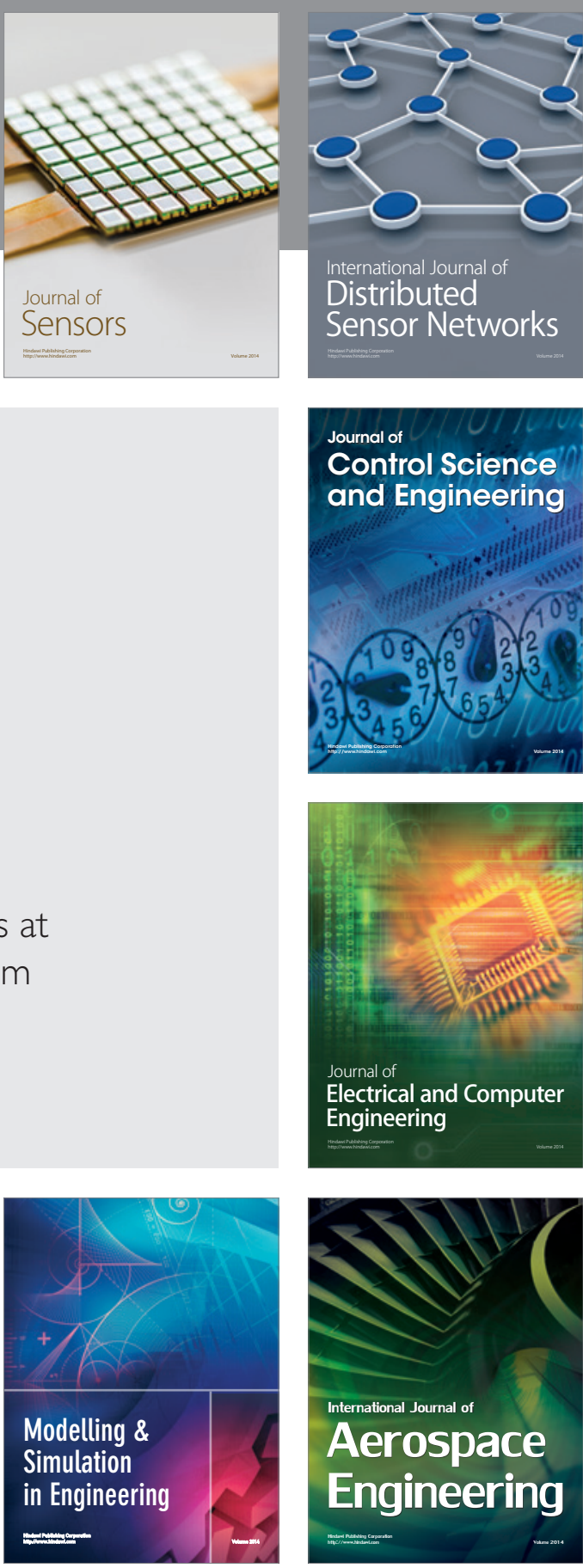

International Journal of

Distributed

Sensor Networks

$-$

Joumal of

Control Science

and Engineering
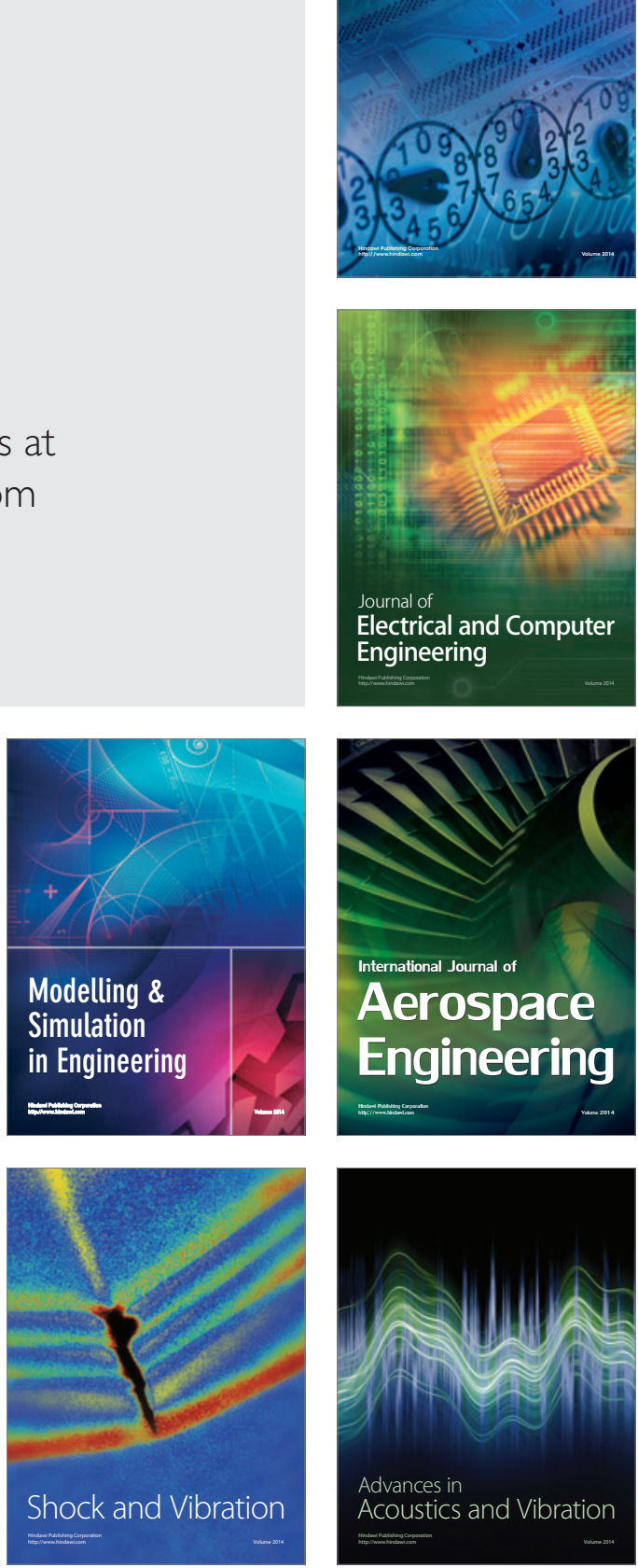